\title{
Direct-imaging discovery of a 12-14 Jupiter-mass object orbiting a young binary system of very low-mass stars ${ }^{\star}$
}

\author{
P. Delorme ${ }^{1}$, J. Gagné ${ }^{2}$, J. H. Girard ${ }^{3}$, A. M. Lagrange ${ }^{1}$, G. Chauvin ${ }^{1}$, M.-E. Naud ${ }^{2}$, D. Lafrenière ${ }^{2}$, R. Doyon ${ }^{2}$, \\ A. Riedel ${ }^{4}$, M. Bonnefoy ${ }^{5}$, and L. Malo \\ 1 UJF-Grenoble 1/CNRS-INSU, Institut de Planétologie et d'Astrophysique de Grenoble (IPAG) UMR 5274, 38041 Grenoble, France \\ e-mail: Philippe.Delorme@obs.ujf-grenoble.fr \\ 2 Département de physique and Observatoire du Mont Mégantic, Université de Montréal, C.P. 6128, Succursale Centre-Ville, \\ Montréal, QC H3C 3J7, Canada \\ 3 European Southern Observatory, Alonso de Córdova 3107, Vitacura, Cassilla 19001, Santiago, Chile \\ ${ }^{4}$ Department of Astrophysics, American Museum of Natural History, Central Park West at 79th Street, New York, NY 10034, USA \\ 5 Max Planck Institute for Astronomy, Königstuhl 17, 69117 Heidelberg, Germany
}

Received 25 January 2013 / Accepted 18 March 2013

\begin{abstract}
Context. Though only a handful of extrasolar planets have been discovered via direct-imaging, each of these discoveries had a tremendous impact on our understanding of planetary formation, stellar formation, and cool atmosphere physics.

Aims. Since many of these newly imaged giant planets orbit massive A or even B stars, we investigate whether giant planets could be found orbiting low-mass stars at large separations.

Methods. We have been conducting an adaptive optic imaging survey to search for planetary-mass companions of young $\mathrm{M}$ dwarfs in the solar neigbourhood, in order to probe different initial conditions of planetary formation.

Results. We report here the direct-imaging discovery of 2MASS J01033563-5515561(AB)b, a 12-14 $M_{\text {Jup }}$ companion at a projected separation of $84 \mathrm{AU}$ from a pair of young late-M stars, with which it shares proper motion. We also detected a Keplerian-compatible orbital motion.

Conclusions. This young L-type object at the planet/brown dwarf mass boundary is the first ever imaged around a binary system at a separation compatible with formation in a disc.
\end{abstract}

Key words. planetary systems - stars: low-mass - methods: observational - instrumentation: adaptive optics

\section{Introduction}

The discovery of hundreds of extrasolar planets in the past 20 years has radically modified our understanding of planetary formation. Though radial velocity and transit detection methods have proven by far the most prolific, the few planetarymass companions that have been discovered by direct-imaging have provided very challenging constraints for formation models, especially the core-accretion model (Pollack et al. 1996) that is preferred for explaining the formation of solar system planets. 2M1207B, discovered by Chauvin et al. (2004) with a mass-ratio of $20-25 \%$, is too massive with respect to its primary to have formed by core accretion, while most of HR8799 (Marois et al. 2008) planets pwould be very difficult to form in situ by core-accretion. Only $\beta$-Pictoris b (Lagrange et al. 2010) fits the standard core-accretion scenario relatively well. Also, several imaged substellar companions (e.g. Chauvin et al. 2005; Lafrenière et al. 2008; Carson et al. 2013) straddle the arbitrary -and debated- $13 M_{\text {Jup }}$ planet/brown dwarf boundary. For most of these massive planets (or light brown dwarfs) the formation mechanism, stellar or planetar, is still being debated (Luhman et al. 2006; Bate 2009; Rafikov 2011; Boss 2011; Stamatellos et al. 2011).

* Based on observations obtained with NACO on VLT UT-4 at ESOParanal (runs 090.C-0698(A) and 70.D-0444(A).
Circumbinary planets, such as Kepler-16 ABb (Doyle et al. 2011), are even rarer, and they provide peculiar constraints on planetary formation scenarios, notably on the influence of binarity on planet-forming discs.

We present here the discovery of 2MASS J01033563$5515561 \mathrm{ABb}$, hereafter $2 \mathrm{MASS} 0103(\mathrm{AB}) \mathrm{b}$, a unique $12-14 M_{\mathrm{Jup}}$ substellar companion to a late $\mathrm{M}$ dwarf binary system.

\section{A $12-14 M_{\text {Jup }}$ companion orbiting a young, late-M binary system}

\subsection{Observations and data reduction}

We imaged 2MASS0103 in November 2012 (run 090.C-0698(A)), in $L^{\prime}$ band as one target of our NACO survey for planetary companions to young nearby $M$ dwarfs (Delorme et al. 2012). We used the infrared wave-front sensor of NACO and observed in pupil-tracking (only $12^{\circ}$ of rotation) and cube mode in $L^{\prime}$. Our follow-up observations in $J H K_{\mathrm{S}}$ on the same night used field-tracking. Table 1 shows the details of our observations.

The target star was resolved as a low contrast, $0.25^{\prime \prime}$ binary, on these raw images and an additional source was identified at $\sim 1.8^{\prime \prime}$ to the north-west of 2MASS0103A. To measure the proper motion of this source, we retrieved ESO archive NACO 

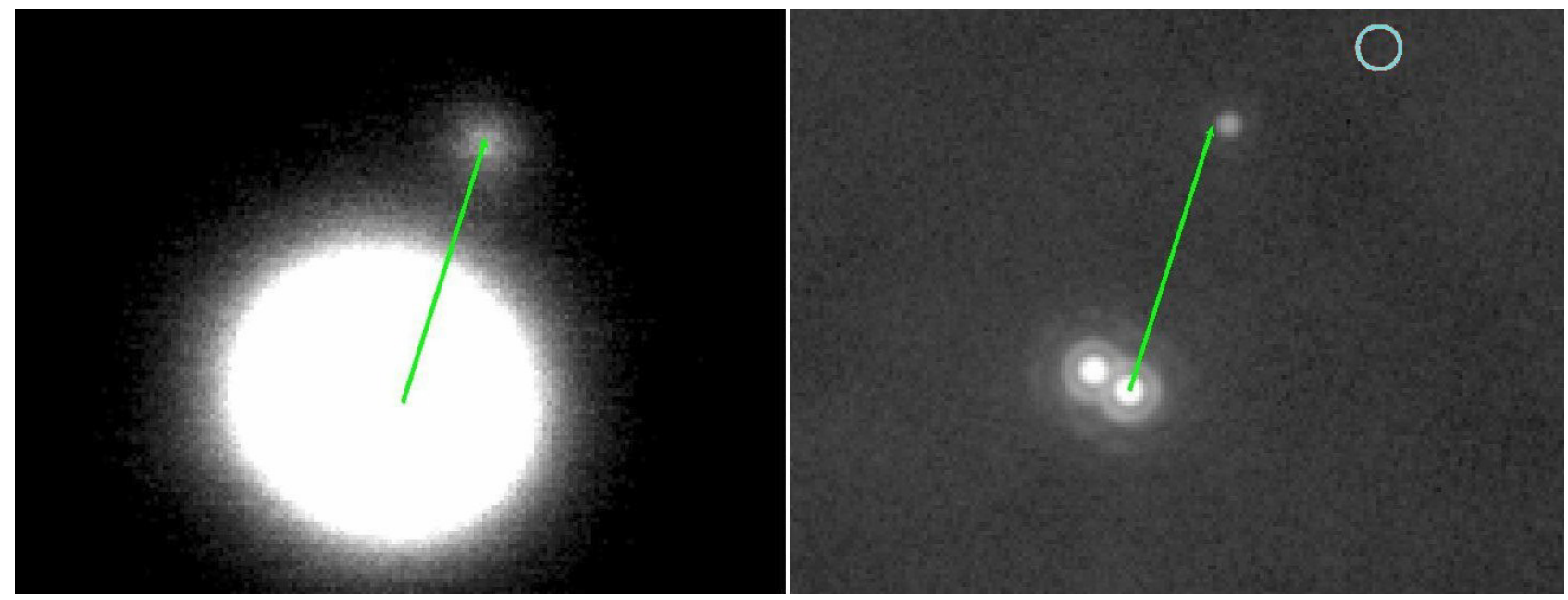

Fig. 1. Left: 2MASS0103(AB)b in October 2002, with NACO in H-band Right: 2MASS0103(AB)b in November 2012, with NACO in $L^{\prime}$ band. The green arrow shows the position of the companion in 2002. The light-blue circle identifies the expected position of the companion if it had been a background source. The host binary was also resolved in 2002, in $H$-band, but this is not visible because of the intensity scale used.

Table 1. Summary of the NACO (VLT-UT4) observations of 2MASS0103AB (RA = 01:03:35.63; Dec $=-55: 15: 56.1)$.

\begin{tabular}{lccc}
\hline \hline UT Date & Filter & Exp. time & Comments \\
\hline \multirow{2}{*}{$2012-11-25$} & $L^{\prime}$ & $32 \times 200 \times 0.2=1280 \mathrm{~s}$ & Seeing: \\
& $K_{\mathrm{S}}$ & $8 \times 20=160 \mathrm{~s}$ & $0.7^{\prime \prime}-0.8^{\prime \prime}$ \\
& $H$ & $8 \times 20=160 \mathrm{~s}$ & Airmass: \\
& $J$ & $4 \times 5=20 \mathrm{~s}$ & $1.16-1.25$ \\
\hline $2002-10-28$ & $H$ & $5 \times 10 \times 2=100 \mathrm{~s}$ & Archive data \\
\hline
\end{tabular}

$H$-band images of 2MASS0103, obtained in October 2002 (run 70.D-0444(A)). These early images were acquired in fieldtracking and with poor adaptive optics correction. We stacked the best $50 \%$ of the frames, for which the central binary was resolved, totalling $100 \mathrm{~s}$ exposure time on target.

We used the IPAG-ADI pipeline as described in Delorme et al. (2012) to reduce the frames (bad pixel interpolation, flat, recentring, derotation, and stacking). Although both the secondary component and the companion clearly appear after a simple stack of all exposures (see Fig. 1), we performed ADI (Marois et al. 2006) and LOCI (Lafrenière et al. 2007) star subtraction procedures to detect other eventual companions. None was detected down to a contrast of $\sim 7.5 \mathrm{mag}$ at $0.5^{\prime \prime}$, resulting in a detection limit of $\sim 2.5 \mathrm{MJup}_{\text {at }}$ a $25 \mathrm{AU}$ for an age of $30 \mathrm{Myr}$ (see discussion below).

\subsection{Host-star properties}

The primary star 2MASS J01033563-5515561 was identified as part of a survey designed to identify new late-type members of the young, nearby moving groups and associations Beta Pictoris, TW Hydrae, Tucana-Horologium (THA), Columba, Carina, Argus and AB Doradus (ABDMG) (Torres et al. 2008). The details of this analysis will be presented in Gagné et al. (in prep.), but the principle is to identify promising candidate members of these moving groups using astrometry, proper motion and photometry from a correlation of 2MASS and WISE catalogues, with a modified version of the Bayesian analysis described in Malo et al. (2013). One of the first robust candidates identified in this project is 2MASS J01033563-5515561, which we have followed with GMOS-S at Gemini South to obtain the optical spectra. This spectrum matches a M5.5/M6 spectral-type and shows strong $\mathrm{H}$-alpha emission at $656 \mathrm{~nm}$, with an equivalent width of $10.23 \pm 0.55 \AA$. No nearby X-ray source was found in the ROSAT archive (Voges et al. 1999), indicating the target is not a strong X-ray emitter. In parallel to this, we have obtained a trigonometric distance of $47.2 \pm 3.1 \mathrm{pc}$ for this object (Riedel, priv. comm., using the CTIO $0.9 \mathrm{~m}$ through the CTIOPI program, using $49 R$-band images taken on 11 nights between October 26, 2007 and November 13, 2012, and reduced using methods from Jao et al. (2005) and Riedel et al. (2011). The complete parallax analysis for 2MASS0103, along with many other objects, will be published in Riedel et al. (in prep.). During the NACO runs described earlier, we also noticed the primary is in fact a binary with a flux ratio of 0.8 in the $L^{\prime}$ band. Taking this binarity and the trigonometric distance into account, we find Bayesian probabilities of $99.6 \%$ and $0.4 \%$ for membership to THA and ABDMG respectively. The field hypothesis has a probability of $10^{-14}$, so 2 MASSO103AB is a strong candidate member of the Tucana-Horologium association, aged $30 \mathrm{Myr}$ (Torres et al. 2008).

We must stress that those probabilities are not absolute ones in the sense that even a sample of candidates with a $100 \%$ Bayesian probability will contain a certain number of false positives. Follow-up observations of robust candidates in Malo et al. (2013) have shown that the false positive rate is $10 \%$ for candidates without parallax in THA. Though the membership analysis in our study is not exactly the same, the risk of a false positive is very low, especially because we do have a parallax measurement, meaning that $2 \mathrm{MASS} 0103 \mathrm{AB}$ is very probably a bona-fide member of THA. We assume in the following that the 2MASS0103 system is aged $30 \mathrm{Myr}$.

According to BT-Settl 2012 isochrones (Allard et al. 2012; Baraffe et al. 2003), and assuming a distance of $47.2 \pm 3.1 \mathrm{pc}$ and an age of $30 \mathrm{Myr}, 2 \mathrm{MASS} 0103 \mathrm{AB}$ is a low-mass binary with masses of $[0.19 ; 0.17] \pm 0.02 M_{\odot}$ for $[\mathrm{A} ; \mathrm{B}]$ respectively, see Table 2. The projected separation between A and B was $0.26 \pm$ $0.01^{\prime \prime}$ in 2002 and $0.249 \pm 0.003^{\prime \prime}$ in 2012 . The projected distance was around $12 \mathrm{AU}$ at both epochs, but the position angle changed significantly, from $71.2^{\circ}$ in 2002 to $61.0^{\circ}$ in 2012 .

\subsection{Proper motion analysis: a bound companion}

During our 2012 November 25, $L^{\prime}$ band NACO observations of 2MASS0103 (run 090.C-0698(A)), we identified a candidate companion with a separation of $1.78^{\prime \prime}$ and a position angle 
Table 2. Host system absolute magnitudes compared with BT-Settl isochrones at $30 \mathrm{Myr}$ absolute magnitudes (2MASS for $J H K$ and NACO for $L^{\prime}$ ).

\begin{tabular}{lcccc}
\hline \hline Filter & $M_{J}$ & $M_{H}$ & $M_{K_{\mathrm{s}}}$ & $M_{L^{\prime}}$ \\
\hline 2M0103A & $7.36 \pm 0.05$ & $6.78 \pm 0.05$ & $6.44 \pm 0.05$ & $6.04^{*}$ \\
2M0103B & $7.56 \pm 0.05$ & $6.98 \pm 0.05$ & $6.64 \pm 0.05$ & $6.24^{*}$ \\
Model 0.2 $M_{\odot}$ & 7.31 & 6.75 & 6.50 & 6.1 \\
$-0.175 M_{\odot}$ & 7.51 & 6.95 & 6.70 & 6.3 \\
\hline
\end{tabular}

Notes. ${ }^{(*)}$ Since no calibrated photometry is available in $L^{\prime}$, these magnitudes are derived from the modelled $K_{\mathrm{S}}-L^{\prime}$.

Table 3. Separation (Sep.) and position angle (PA) of the companion, with respect to $2 \mathrm{MASS} 0103 \mathrm{~A}$ and to the centre of mass of the binary.

\begin{tabular}{lcc}
\hline \hline & $2002-10-28$ & $2012-11-25$ \\
\hline Sep. from 2M0103A (") & $1.682 \pm 0.015$ & $1.784 \pm 0.003$ \\
PA from 2M0103A $\left(^{\circ}\right)$ & $341.7 \pm 0.05^{*}$ & $339.8 \pm 0.01^{*}$ \\
\hline Sep. from barycenter (") & $1.718 \pm 0.015$ & $1.770 \pm 0.003$ \\
PA from barycenter $\left(^{\prime \prime}\right)$ & $338.0 \pm 0.05^{*}$ & $336.1 \pm 0.01^{*}$ \\
\hline
\end{tabular}

Notes. ${ }^{(*)}$ The error in position angle refers to the relative error between both epochs. The absolute error, dominated by systematic uncertainties in the position of the reference stars in theta Ori, is $\pm 0.4^{\circ}$.

of $339.3^{\circ}$ from the primary 2MASS0103A. Even if contamination by background objects is relatively low in $L^{\prime}$ band compared to shorter wavelength (see Delorme et al. 2012), the probability that this companion was a contaminant was high. However, the companion was redder in $K_{\mathrm{S}}-L^{\prime}$ than its late $\mathrm{M}$ host system, meaning that the companion is an even later spectral type, considerably decreasing the likelihood of the contaminant hypothesis, but not incontrovertibly proving that the companion is bound. A definite proof of companionship was however provided thanks to archive images taken with NACO on October 28, 2002. Using this ten years time base, we could determine that $2 \mathrm{MASS} 0103(\mathrm{AB}) \mathrm{b}$ unambiguously (contamination probability $<0.001 \%$, taking parallax motion into account) shares the proper motion of 2MASS0103AB (see Fig. 1) and is therefore a bound companion. The relative astrometry at each epoch is shown in Table 3 .

\subsection{Companion properties}

Our current information about the physical properties of the system relies on the $J, H, K$ and $L^{\prime}$ photometry and on astrometry from our 2012 November NACO run, as well as 2002 October NACO archive images. The resulting Moffat-fitting photometry is $J=15.4 \pm 0.3, H=14.2 \pm 0.2, K_{\mathrm{S}}=13.6 \pm 0.2$, and $L^{\prime}=12.6 \pm 0.1$. We emphasize that all these measurements were derived relative to $2 \mathrm{MASS} 0103 \mathrm{~A}$, which introduces significant systematic errors (hence the large errors bars). The absolute magnitudes of the companion, for a distance of $47.2 \mathrm{pc}$, are shown in Table 4 and are compatible with 2MASS0103(AB)b being a 12 to $14 M_{\text {Jup }}$ companion orbiting at $84 \mathrm{AU}$ around the young low-mass binary $2 \mathrm{MASS} 0103 \mathrm{AB}$. The very red colours of 2MASS0103(AB)b in $J H K$ s do not match the colours of field objects of similar absolute magnitudes and are similar to known, early L-type, young planetary-mass objects, thus independently confirming the youth of the system (see also Fig. 2).

It should be noted that the age of THA is not perfectly known, and the dispersion of the age estimations of individual
Table 4. Companion absolute magnitudes compared with BT-Settl isochrones at $30 \mathrm{Myr}$ and $5 \mathrm{Gyr}$ (field hypothesis) absolute magnitudes, and other known companions at the planet/brown dwarf mass boundary.

\begin{tabular}{l|cccc}
\hline \hline Companions & $M_{J}$ & $M_{H}$ & $M_{K \mathrm{~s}}$ & $M_{L^{\prime}}$ \\
\hline $2 \mathrm{M} 0103 \mathrm{ABb}$ & $12.1 \pm 0.3$ & $10.9 \pm 0.2$ & $10.3 \pm 0.2$ & $9.3 \pm 0.1$ \\
$\kappa$ And b & $12.7 \pm 0.3$ & $11.7 \pm 0.2$ & $11.0 \pm 0.4$ & $9.5 \pm 0.1$ \\
$\mathrm{AB}$ pic b & $12.9 \pm 0.1$ & $11.4 \pm 0.1$ & $10.8 \pm 0.1$ & $9.9 \pm 0.1$ \\
\hline Models & & & & \\
$12 M_{\text {Jup }}$ & 12.8 & 11.9 & 11.3 & 10.0 \\
$15 M_{\text {Jup }}$ & 10.9 & 10.3 & 9.9 & 9.0 \\
Field & 10.8 & 10.3 & 10.0 & 9.4 \\
\hline
\end{tabular}

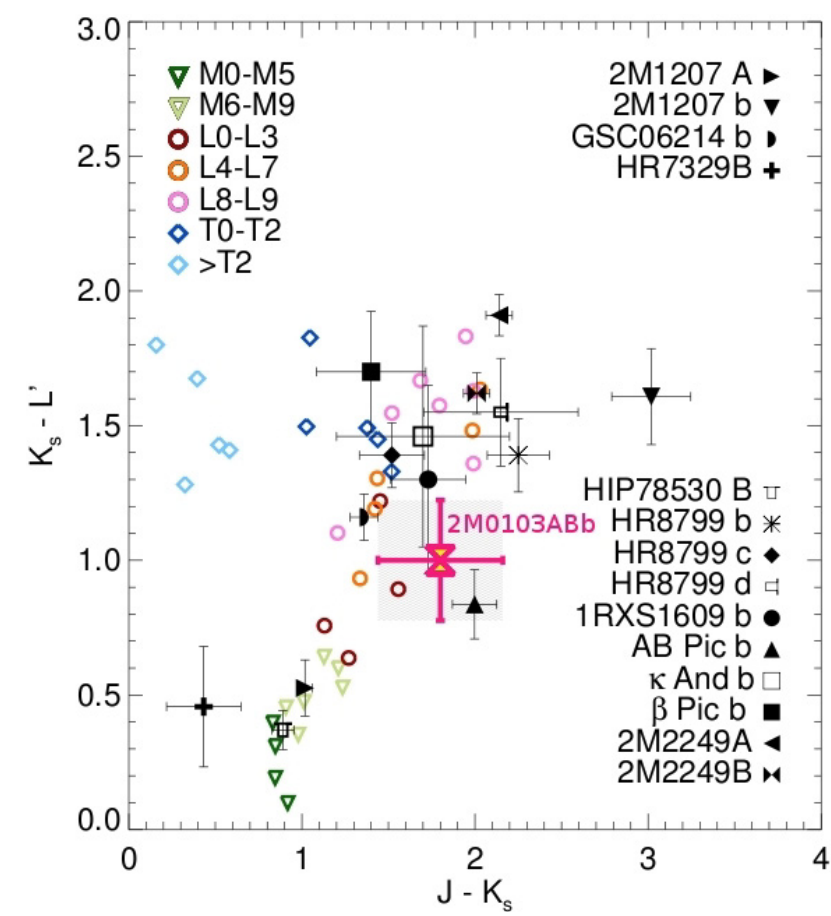

Fig. 2. $J-K_{\mathrm{S}}$ versus $K_{\mathrm{S}}-L$ colour-colour diagram showing 2MASS0103(AB)b, together with other known planetary and brown dwarf companions to young stars (taken from Bonnefoy et al. 2013; Bailey et al. 2013). The symbols without error bars show the colour of field M, L, and T dwarfs (taken from Golimowski et al. 2004).

stars in THA spans the 20-50 Myr range (Zuckerman \& Webb 2000; Torres et al. 2000). If we assume an age of $20 \mathrm{Myr}$, 2MASS0103(AB)b would be a 12-13 $M_{\text {Jup }}$ planet, while it would be a 14-15 $M_{\text {Jup }}$ brown dwarf if we assume an age of 50 Myr. In spite of the name change, the physical differences in mass estimates for the $20 \mathrm{Myr}$ and the $50 \mathrm{Myr}$ hypothesis are much smaller than those derived in Marois et al. (2010) for HR8799bcde planets in the same age range. An explanation is that objects that are more massive than $\sim 10 M_{\text {Jup }}$ undergo some deuterium burning in this age range, somewhat compensating for cooling-down mechanisms. However, since there is currently no robust independent mass constraint for any imaged exoplanet (saved to some extent for $\beta$ Pic b, see Lagrange et al. 2012), it is probable that the systematic uncertainties coming from substellar model inaccuracies are larger than those arising from age uncertainties. For the sake of comparison with other substellar companions found in associations of the same mean age of $30 \mathrm{Myr}$, we assume in the following that 2MASS0103(AB)b is a $12-14 M_{\text {Jup }}$ object aged $30 \mathrm{Myr}$.

A possible analogue, if confirmed as bound, would be the substellar object located at a projected separation of 1100 AU 


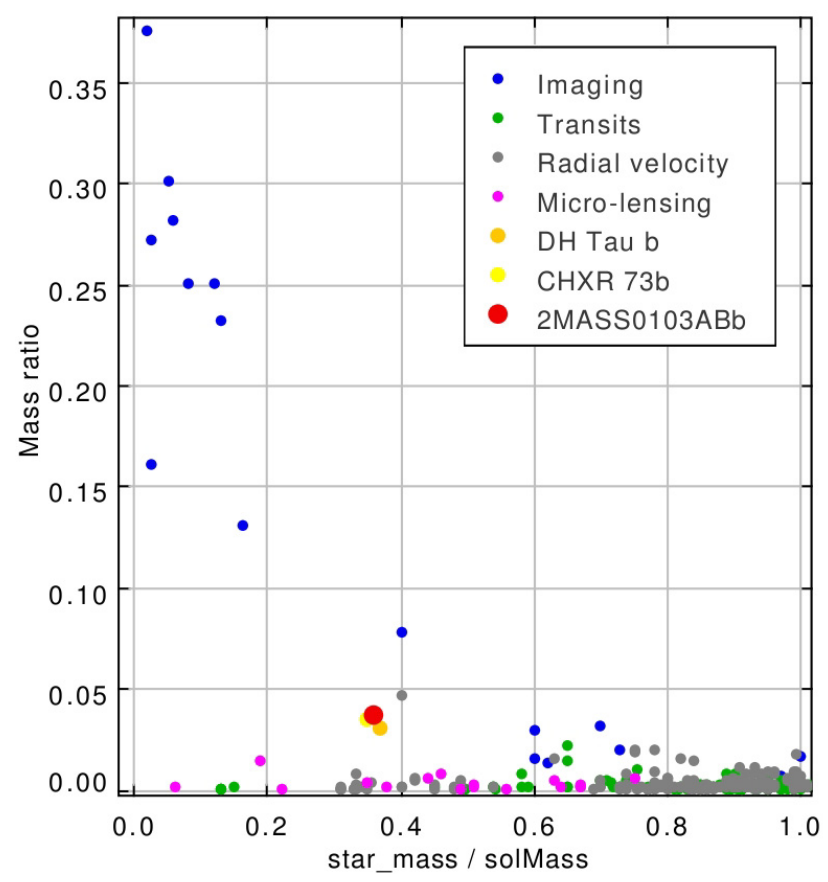

Fig. 3. Substellar companion to stellar host mass ratio versus stellar host mass, sorted by their discovery technique, from the exoplanet.eu database (Schneider et al. 2011).

from the binary system SR12AB (Kuzuhara et al. 2011). The properties of 2MASS0103(AB)b (mass of 12-14 $M_{\mathrm{Jup}}$, age of $30 \mathrm{Myr}$, colours, projected separation of $>50 \mathrm{AU}$ ), and observed colours are also much like $\mathrm{AB}$ pic b (K1V Chauvin et al. 2005) or $\kappa$ Andromeda b (Carson et al. 2013). The properties of the host systems are, however, quite different. While $\kappa$ Andromeda is a massive B star $\left(\sim 2.5 M_{\odot}\right.$, mass ratio of $\sim 0.5 \%$ ), $2 \mathrm{MASS} 0103 \mathrm{AB}$ is a close binary system composed of two late $\mathrm{M}$ dwarfs, whose combined mass is $\sim 0.36 M_{\odot}$, resulting in a mass ratio of approximatively $3.6 \%$ for the system.

The position of the companion at each epoch was derived by Moffat-fitting, and the orientation of the detector was calibrated using NACO calibration images of theta Ori obtained close to the time of the science images. As shown in Table 3, the relative astrometry is accurate enough to detect the orbital motion of the companion around the centre of mass of the system, with a projected motion of $77 \pm 15$ mas over ten years. The corresponding velocity at $47.2 \mathrm{pc}$ is $1.7 \pm 0.3 \mathrm{~km} \mathrm{~s}^{-1}$. The Keplerian velocity, assuming a circular orbit of $84 \mathrm{AU}$ around the $0.36 M_{\odot}$ system is $1.96 \mathrm{~km} \mathrm{~s}^{-1}$, corresponding to a period of 1280 years and is fully compatible with our measurement. It is to be noted that the secondary and the companion rotate in the same direction and that their observed orbital motion can be compatible with a faceon orbit but not with an edge-on one.

\section{A challenge for stellar and planetary formation theories}

The mass ratio between $2 \mathrm{MASS} 0103(\mathrm{AB}) \mathrm{b}$ and its host system is $\sim 0.036$, which is too low to match known low mass multiple systems (See Fig. 3 and also Allers et al. 2007), but still higher than most star-planet systems confirmed so far. Systems with similar mass ratios, but ones that are almost one order of magnitude more massive with 55-95 $M_{\text {Jup }}$ brown dwarfs or very lowmass stars orbiting massive $\left(1.2-2 M_{\odot}\right)$ stars, have been identified by Janson et al. (2012). In case formation mechanisms scaled simply with mass, the same processes could be at work to explain the formation of these very different kinds of objects. If we keep to the more similar low-mass star systems shown in Fig. 3, the mass ratio of $2 \mathrm{MASS} 0103(\mathrm{AB}) \mathrm{b}$ is very close to those of DH tau B (8-22 $M_{\text {Jup }}$, separation of $330 \mathrm{AU}$ ) and CHXR 73b (7-20 $M_{\text {Jup }}$, separation of $210 \mathrm{AU}$ ) (Itoh et al. 2005; Luhman et al. 2006), but its projected separation is much smaller. Luhman et al. (2006) state that neither DH tau B nor CHXR 73b could be formed in situ by core accretion or disc instability because of the very large separation from their host-stars, and the same holds for the $1100 \mathrm{AU}$ candidate companion to SR12AB (Kuzuhara et al. 2011). The case is different for 2MASS0103(AB)b, at a separation of only 84 AU. At such separations, a formation in a gravitationally unstable primordial circumbinary disk would be fully compatible with planetary formation by gravitational instabilities, as described by Boss (2011). However, this scenario is being discussed: Dodson-Robinson et al. (2009) claim that objects formed by disc instabilities around M-dwarfs should have $\sim 10 \%$ of the mass of the host system, meaning that 2MASS0103(AB)b would not be massive enough for such a scenario. Other studies (Rafikov 2009; Stamatellos et al. 2011) find that such low-mass discs cannot fragment at all. Simultaneous formation and ejection of the three components in the massive disc of a more massive orginal host-star is plausible, in a scenario akin to what is described in Stamatellos \& Whitworth (2009), but the central binary components with masses of 0.17 and $0.19 M_{\odot}$ are more massive than most objects formed in Stamatellos \& Whitworth (2009) simulations.

A planetary formation scenario by core accretion (e.g. Kennedy \& Kenyon 2008; Mordasini et al. 2009; Rafikov 2011) can very probably be excluded for several reasons. First, the separation is too large for a formation in situ. Second, the companion has $\sim 3.6 \%$ of the mass of its host system, which is the same order of magnitude as the maximum total mass of the protoplanetary disc from which core accretion planets are formed. Finally, such a 12-14 $M_{\text {Jup }}$ companion would be a very rare occurrence, according to the core accretion planetary mass-function derived by Mordasini et al. (2012).

A purely stellar formation mode by turbulent core fragmentation (see e.g. Padoan \& Nordlund 2002; Bate 2009; Hennebelle \& Chabrier 2011) is plausible, and in this case 2MASS0103(AB)b would be an extreme case of hierarchical triple stellar with a third component in the 12-14 $M_{\text {Jup }}$ mass range. However, a stellar formation scenario would require that cores can naturally fragment into such low-mass objects, without requiring any ejection from the the accretion reservoir (such as described in Reipurth \& Clarke 2001; Bate \& Bonnell 2005), because it would be difficult to starve the accretion of the third component without also stopping accretion on the central binary. From hydrodynamical simulations of stellar formation by cloud fragmentation, Bate (2012) claims that "brown dwarfs with masses $<15 M_{\text {Jup }}$ should be very rare", implying that formation by direct core fragmentation of a $12-14 M_{\text {Jup }}$ object, such as 2MASS0103(AB)b, would be possible but uncommon.

The discovery of 2MASS0103(AB)b stretches most current stellar and planetary formation theories to their limits, while others, such as core-accretion, can probably be excluded. The very existence of such a peculiar system therefore provides a very valuable test case against which current and future stellar and planetary formation theoretical models can be tested.

Acknowledgements. We acknowledge support from the French National Research Agency (ANR) through the GuEPARD project grant ANR10BLANC0504-01. We acknowledge financial support from the "Programme National de Physique Stellaire” (PNPS) of CNRS/INSU, France. 
P. Delorme et al.: Direct-imaging of a $12-14 M_{\text {Jup }}$ object orbiting a M-dwarf binary system

\section{References}

Allard, F., Homeier, D., \& Freytag, B. 2012, Roy. Soc. London Philos. Trans. Ser. A, 370, 2765

Bailey, V., Hinz, P. M., Currie, T., et al. 2013, ApJ, 767, 31

Baraffe, I., Chabrier, G., Barman, T. S., Allard, F., \& Hauschildt, P. H. 2003, A\&A, 402, 701

Bate, M. R. 2009, MNRAS, 392, 590

Bate, M. R. 2012, MNRAS, 419, 3115

Bate, M. R., \& Bonnell, I. A. 2005, MNRAS, 356, 1201

Bonnefoy, M., Boccaletti, A., Lagrange, A.-M., et al. 2013, A\&A, in press, DOI: $10.1051 / 0004-6361 / 201220838$

Boss, A. P. 2011, ApJ, 731, 74

Carson, J., Thalmann, C., Janson, M., et al. 2013, ApJ, 763, L32

Chauvin, G., Lagrange, A.-M., Dumas, C., et al. 2004, A\&A, 425, L29

Chauvin, G., Lagrange, A.-M., Zuckerman, B., et al. 2005, A\&A, 438, L29

Delorme, P., Lagrange, A. M., Chauvin, G., et al. 2012, A\&A, 539, A72

Dodson-Robinson, S. E., Veras, D., Ford, E. B., \& Beichman, C. A. 2009, ApJ, 707, 79

Doyle, L. R., Carter, J. A., Fabrycky, D. C., et al. 2011, Science, 333, 1602

Golimowski, D. A., Leggett, S. K., Marley, M. S., et al. 2004, AJ, 127, 3516

Hennebelle, P., \& Chabrier, G. 2011, ApJ, 743, L29

Itoh, Y., Hayashi, M., Tamura, M., et al. 2005, ApJ, 620, 984

Janson, M., Jayawardhana, R., Girard, J. H., et al. 2012, ApJ, 758, L2

Jao, W.-C., Henry, T. J., Subasavage, J. P., et al. 2005, AJ, 129, 1954

Kennedy, G. M., \& Kenyon, S. J. 2008, ApJ, 673, 502

Kuzuhara, M., Tamura, M., Ishii, M., et al. 2011, AJ, 141, 119

Lafrenière, D., Marois, C., Doyon, R., Nadeau, D., \& Artigau, É. 2007, ApJ, 660,770
Lafrenière, D., Jayawardhana, R., \& van Kerkwijk, M. H. 2008, ApJ, 689, L153 Lagrange, A.-M., Bonnefoy, M., Chauvin, G., et al. 2010, Science, 329, 57 Lagrange, A.-M., De Bondt, K., Meunier, N., et al. 2012, A\&A, 542, A18

Luhman, K. L., Wilson, J. C., Brandner, W., et al. 2006, ApJ, 649, 894

Malo, L., Doyon, R., Lafrenière, D., et al. 2013, ApJ, 762, 88

Marois, C., Lafrenière, D., Doyon, R., Macintosh, B., \& Nadeau, D. 2006, ApJ, 641, 556

Marois, C., Macintosh, B., Barman, T., et al. 2008, Science, 322, 1348

Marois, C., Macintosh, B., \& Véran, J.-P. 2010, in SPIE Conf. Ser., 7736

Mordasini, C., Alibert, Y., \& Benz, W. 2009, A\&A, 501, 1139

Mordasini, C., Alibert, Y., Benz, W., Klahr, H., \& Henning, T. 2012, A\&A, 541, A97

Padoan, P., \& Nordlund, Å. 2002, ApJ, 576, 870

Pollack, J. B., Hubickyj, O., Bodenheimer, P., et al. 1996, Icarus, 124, 62

Rafikov, R. R. 2009, ApJ, 704, 281

Rafikov, R. R. 2011, ApJ, 727, 86

Reipurth, B., \& Clarke, C. 2001, AJ, 122, 432

Riedel, A. R., Murphy, S. J., Henry, T. J., et al. 2011, AJ, 142, 104

Schneider, J., Dedieu, C., Le Sidaner, P., Savalle, R., \& Zolotukhin, I. 2011, A\&A, 532, A79

Stamatellos, D., \& Whitworth, A. P. 2009, MNRAS, 392, 413

Stamatellos, D., Maury, A., Whitworth, A., \& André, P. 2011, MNRAS, 413, 1787

Torres, C. A. O., da Silva, L., Quast, G. R., de la Reza, R., \& Jilinski, E. 2000, AJ, 120, 1410

Torres, C. A. O., Quast, G. R., Melo, C. H. F., \& Sterzik, M. F. 2008, Young Nearby Loose Associations, ed. B. Reipurth, 757

Voges, W., Aschenbach, B., Boller, T., et al. 1999, A\&A, 349, 389

Zuckerman, B., \& Webb, R. A. 2000, ApJ, 535, 959 